P.J.S. Gonçalves, L.F. Mendonça, J. M C Sousa, J.R.C. Pinto (2008)

\title{
Uncalibrated Eye-to-Hand Visual Servoing Using Inverse Fuzzy Models.
}

IEEE Transactions on Fuzzy Systems, 16 (2) pp. 341-353.

http://ieeexplore.ieee.org/document/4374115/

\section{Abstract}

A new uncalibrated eye-to-hand visual servoing based on inverse fuzzy modeling is proposed in this paper. In classical visual servoing, the Jacobian plays a decisive role in the convergence of the controller, as its analytical model depends on the selected image features. This Jacobian must also be inverted online. Fuzzy modeling is applied to obtain an inverse model of the mapping between image feature variations and joint velocities. This approach is independent from the robot's kinematic model or camera calibration and also avoids the necessity of inverting the Jacobian online. An inverse model is identified for the robot workspace, using measurement data of a robotic manipulator. This inverse model is directly used as a controller. The inverse fuzzy control scheme is applied to a robotic manipulator performing visual servoing for random positioning in the robot workspace. The obtained experimental results show the effectiveness of the proposed control scheme. The fuzzy controller can position the robotic manipulator at any point in the workspace with better accuracy than the classic visual servoing approach.

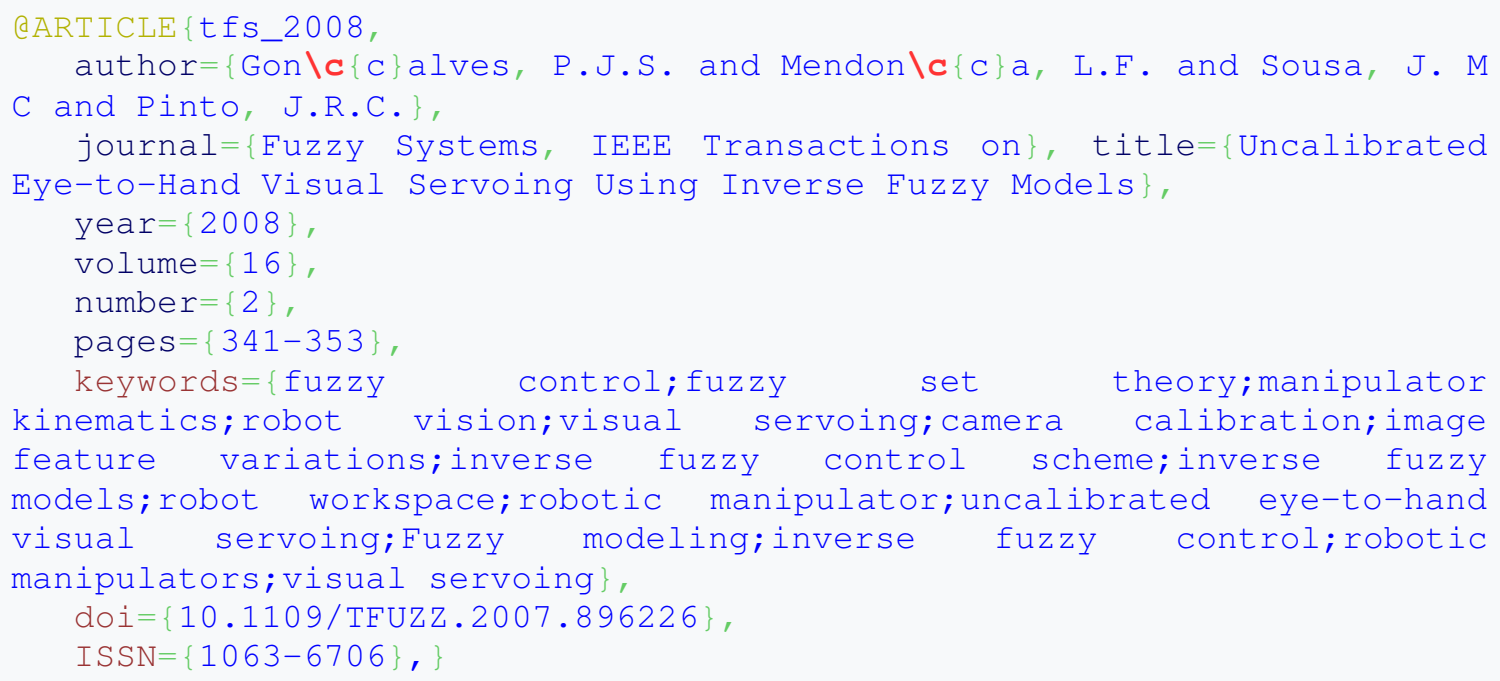

